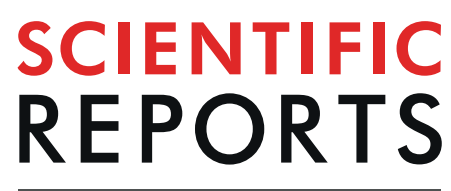

natureresearch

\title{
Expansion of LINEs and species- specific DNA repeats drives genome expansion in Asian Gypsy Moths
}

\author{
Francois Olivier Hebert $\mathbb{C}^{1 *}$, Luca Freschi ${ }^{1}$, Gwylim Blackburn ${ }^{1}$, Catherine Béliveau ${ }^{2}$, \\ Ken Dewar ${ }^{3}$, Brian Boyle ${ }^{1}$, Dawn E. Gundersen-Rindal ${ }^{4}$, Michael E. Sparks ${ }^{4}$, Michel Cusson $\mathbb{D}^{1,2}$, \\ Richard C. Hamelin ${ }^{1,5}$ \& Roger C. Levesque $\mathbb{B}^{1}$
}

Two subspecies of Asian gypsy moth (AGM), Lymantria dispar asiatica and L. dispar japonica, pose a serious alien invasive threat to North American forests. Despite decades of research on the ecology and biology of this pest, limited AGM-specific genomic resources are currently available. Here, we report on the genome sequences and functional content of these AGM subspecies. The genomes of $L$.d. asiatica and L.d. japonica are the largest lepidopteran genomes sequenced to date, totaling 921 and 999 megabases, respectively. Large genome size in these subspecies is driven by the accumulation of specific classes of repeats. Genome-wide metabolic pathway reconstructions suggest strong genomic signatures of energy-related pathways in both subspecies, dominated by metabolic functions related to thermogenesis. The genome sequences reported here will provide tools for probing the molecular mechanisms underlying phenotypic traits that are thought to enhance AGM invasiveness.

North American forests face unprecedented threats from multiple Forest Invasive Alien Species (FIAS) that can potentially cause large-scale disturbances and major biological, social, and economic impacts. One of the most threatening FIAS currently identified in Canada and the United States is the Asian gypsy moth (AGM). The term AGM refers to a group of closely related species of Lymantria moths (order Lepidoptera), including $L$. dispar asiatica, L. dispar japonica, L. umbrosa, L. albescens, and L. postalba ${ }^{1-3}$. Although the European gypsy moth (EGM; Lymantria dispar dispar) became established in North America more than 100 years ago, AGM are currently considered "non-established". However, AGM represent a constant threat as they have often been intercepted at North American ports. AGM also exhibit several ecological characteristics that may increase their invasive capacity compared to EGM. First, they have a broad host range ( $>500$ botanical species), including more coniferous species than $\mathrm{EGM}^{4}$. Second, they are attracted to light, which makes them more inclined to oviposit on cargos and ships that transport goods around the world ${ }^{5}$. Third, eggs of AGM subspecies require shorter chilling time before hatching ${ }^{6,7}$. As a result, increasing temperatures due to climate change may help spread AGM subspecies into new geographic regions. Fourth, unlike EGM, AGM females are capable of direct and sustained flight and so can disperse on a larger spatial scale ${ }^{5,8}$. Overall, these features likely enhance AGM invasion capacity. Notably, AGM and EGM can also successfully interbreed, producing flight-capable and fertile hybrid progeny ${ }^{9}$. Monitoring the large-scale dispersal patterns of these insects around the world could help reduce their impact on forest ecosystems.

The ability to predict gypsy moth female invasiveness is of critical importance in developing and implementing adequate and efficient management programs. Yet, despite considerable research efforts invested in understanding behavioral and ecological aspects of Lymantria moths, the molecular mechanisms responsible for the expression of key invasive traits in this species complex remain largely uncharacterized. Only partial transcriptome sequences are available for $\mathrm{EGM}^{10}$ and L.d. asiatica ${ }^{11,12}$. Although a draft genome sequence was recently published for $\mathrm{EGM}^{13}$, no whole genome sequences currently exist for any AGM subspecies.

\footnotetext{
${ }^{1}$ Institut de Biologie Intégrative et des Systèmes (IBIS), Université Laval, Ville de Québec, Canada. ${ }^{2}$ Laurentian Forestry Centre, Canadian Forest Service, Natural Resources Canada, Quebec City, Quebec, Canada. ${ }^{3}$ Department of human genetics, McGill University, Montreal, Quebec, Canada. ${ }^{4}$ United States Department of Agriculture ARS Invasive Insect Biocontrol and Behavior Laboratory, Beltsville, Maryland, USA. ${ }^{5}$ Department of Forest and Conservation Sciences, Faculty of Forestry, University of British Columbia, Vancouver, British Columbia, Canada. *email: francois-olivier.gagnon-hebert.1@ulaval.ca
} 


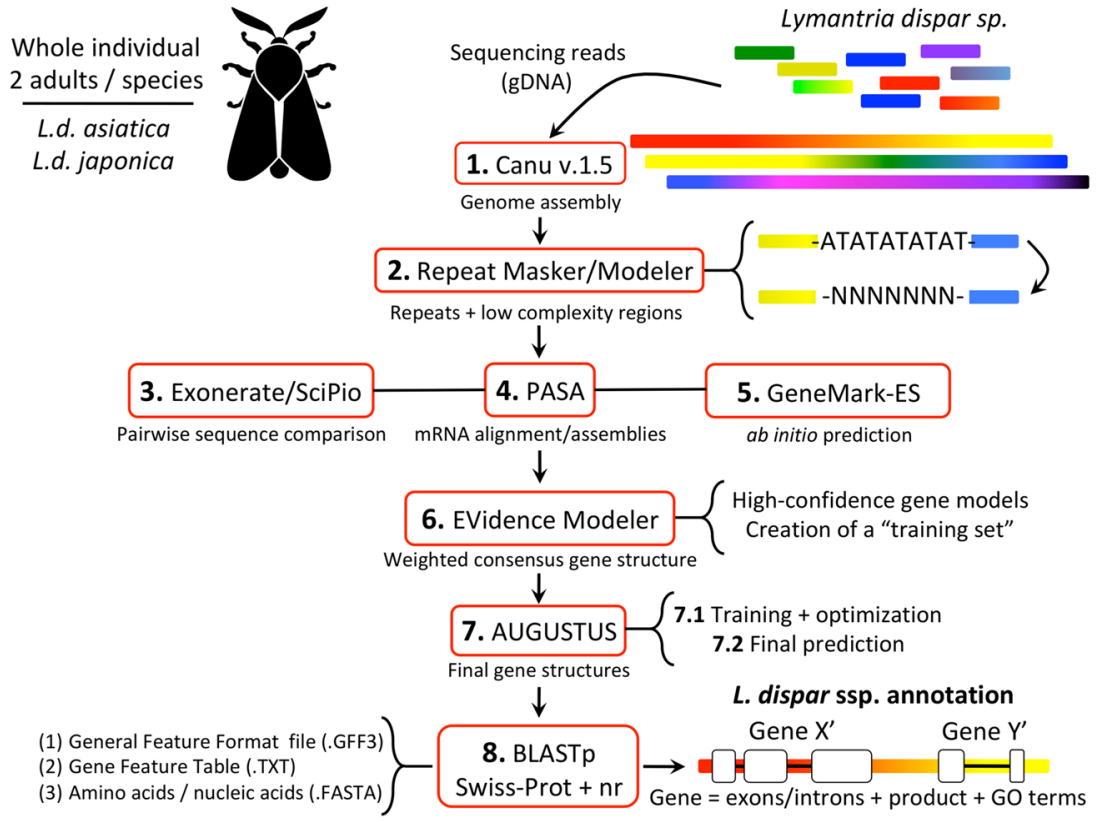

Figure 1. Genome annotation pipeline specifically optimized and trained to identify gene structures in Lepidopteran genomes.

Here we present gypsy moth reference genomes for L.d. asiatica and L.d. japonica, and we describe their gene content and organization. We show that these AGM subspecies possess the largest genomes sequenced to date among lepidopterans, a characteristic that can be explained by the accumulation of numerous transposable elements. The reference genomes reported here provide valuable genomic information that can be used for gene mapping, comparative analyses, development of genome engineering tools, as well as to better understand key aspects related to the biological characteristics that make AGM one of the greatest threats to North American forests.

\section{Results and Discussion}

Largest sequenced lepidopteran genome. Through our in-house assembly and annotation pipeline (Fig. 1, see Supplementary Information Section S2), we sequenced the genome of L.d. asiatica using DNA extracted from two adult males, with 70 PacBio RSII SMRT cells and the P6-C4 chemistry. Our pipeline was trained and optimized to specifically annotate moth genomes (Fig. 1, step 7.1 - Augustus training). This yielded 8.5 million raw sequencing reads for a total of $35.6 \mathrm{~Gb}$ sequenced, with uncorrected genome coverage of $36 \times$ (CANU assembly report). Similarly, DNA from two adult L.d. japonica males was used to generate 44 PacBio RSII SMRT cells (also with P6-C4 chemistry), yielding 5.4 million raw sequencing reads, for a total of $30.6 \mathrm{~Gb}$ sequenced, with uncorrected genome coverage of $31 \times$ (CANU assembly report). Post-processing steps returned total genome sizes of $921 \mathrm{Mb}$ (final coverage $=47 \mathrm{X}$ ) and $999 \mathrm{Mb}$ (final coverage $=37 \mathrm{X}$ ), with N50s of $212 \mathrm{~Kb}$ and $137 \mathrm{~Kb}$, for L.d. asiatica and L.d. japonica, respectively (Table 1, see Supplementary Information Sections S2 and S3). Previous work on genome sizes in the Lepidoptera established that the total genome size of L. dispar dispar (EGM) was $\sim 1,007 \mathrm{Mb}^{14}$. Additional flow cytometry-based EGM genome size determinations generated comparable results (male genome size of $993.3 \pm 6.2 \mathrm{Mb}$ and female genome size of $983.2 \pm 6.7 \mathrm{Mb}$; work conducted in the laboratory of Dr. Spencer Johnston, Texas A\&M University, United States). This result provides indirect corroboration for our genome size estimates in AGM and suggests a high level of completeness for the AGM assemblies. A k-mer distribution analysis performed on both genomes revealed that eight percent (L.d. asiatica) and 10 percent (L.d. japonica) of the k-mers that we generated were present in more than one copy, which suggests a low level of potential allelic contigs in our assemblies (see Supplementary Information Section S3 and Supplementary Fig. S1). The total genome sizes and low level of allelic duplicates indicate that the genomes generated in this study can be considered as subspecies-level references. This will ensure that each reference genome contains the least amount of errors. The $78 \mathrm{Mb}$ difference between L.d. asiatica and L.d. japonica genome sizes may be explained in part by significant differences in raw sequencing read lengths in each of the two independent sequencing and assembly procedures. Corrected read length distributions showed that L.d. japonica had a read N50 of 11.9 $\mathrm{Kb}$, while L.d. asiatica had a read N50 of $9.4 \mathrm{~Kb}$ (Supplementary Fig. S2), which could explain why we obtained a larger genome assembly in the case of L.d. japonica. In addition, more Arthropoda BUSCO (Benchmarking Universal Single Copy Orthologs ${ }^{15}$ ) genes were captured in the L.d. japonica assembly ( $98.2 \%$ total BUSCO coverage) compared to that of L.d. asiatica ( $96.5 \%$ total BUSCO coverage), consistent with the observation that the L.d. japonica genome is larger and closer to the expected genome size than the L.d. asiatica genome (Supplementary Information Section S2). These results expand the range of reported genome assembly sizes of related lepidopteran species such as the urticating pine defoliator (Thaumetopoea pityocampa, $537 \mathrm{Mb}^{16}$ ), the silk worm (Bombyx 


\begin{tabular}{|l|l|l|l|l|}
\hline Feature & Bmo* $^{*}$ & Ldd $^{\square}$ & Lda & Ldj \\
\hline Total size $(\mathrm{Mb})$ & 481 & 865 & 921 & 999 \\
\hline Contigs & 88,637 & 194,709 & 8,190 & 11,303 \\
\hline Scaffolds & 43,463 & 134,446 & n.a. & n.a. \\
\hline N50 (Kb) & 4,008 & 5,068 & 212 & 137 \\
\hline N90 (Kb) & 0.262 & 0.245 & 73 & 82 \\
\hline Repeat (\%) & 43.6 & 36 & 59.9 & 59.6 \\
\hline GC content (\%) & 37.7 & 35.2 & 38.7 & 38.5 \\
\hline Protein-coding genes & 15,488 & 13,331 & 19,588 & 23,292 \\
\hline Exon (\%) & 4.7 & 1.8 & 2.7 & 3.0 \\
\hline Intron (\%) & 16.1 & 17 & 27.6 & 28.4 \\
\hline BUSCO coverage (\%) A & 95.5 & 89.2 & 96.5 & 98.2 \\
\hline
\end{tabular}

Table 1. Composition of the two AGM Lymantria dispar spp. genomes sequenced in this study in comparison to the sequenced genomes of EGM Lymantria dispar dispar and the well characterized sister species Bombyx mori. Abbreviations: Bmo, Bombyx mori; Ldd, Lymantria dispar dispar; Lda, Lymantria dispar asiatica; Ldj, Lymantria dispar japonica. *Reference genome ASM15162v1, accession number GCA_000151625.1 (http://

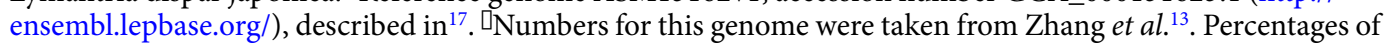
exon and introns for Bmo and Ldd were taken from this reference, while the values for Lda and Ldj were calculated based on the GFF3 files produced in this study. A BUSCO values were obtained form BUSCO v.3.0 ${ }^{15}$. Reported values correspond to total BUSCO genes retrieved, i.e. complete (single + duplicated) and fragmented.

mori, $481 \mathrm{Mb}^{17}$ ), the greater wax moth (Galleria mellonella, $578 \mathrm{Mb}^{18}$ ), the Japanese oak silk moth (Antheraea yamamai, $656 \mathrm{Mb}^{19}$ ), the common wood-white moth (Leptidae sinapis, $643 \mathrm{Mb}^{20}$ ), and the red-banded hairstreak (Calycopis cecrops, $729 \mathrm{Mb}$, according to http://lepbase.org ${ }^{21}$ ). The largest lepidopteran genome sequenced before this study was the genome of the closely related EGM (Lymantria dispar dispar, $865 \mathrm{Mb}^{13}$ ), making the AGM genomes obtained here the largest lepidopteran genomes sequenced to date.

Gene sets exhibited numbers of protein-coding genes within the range found in other Lepidopteran genomes (between 10,117 and 29,902 genes, see Supplementary Table S3 for details). Genome assembly post-processing steps, focused on the reduction of redundancy through sequence similarity, allowed us to identify the structure of 19,588 and 23,292 genes in L.d. asiatica and L.d. japonica respectively (Fig. 2a, Supplementary Information Section S3). Similar proportions of KEGG KO categories identified between the two genomes suggest that they are very similar in terms of overall functional content (Supplementary Fig. S3). While the difference in the numbers of genes we identified in the two subspecies was unexpectedly high, the greater value obtained for L.d. japonica may be attributable (at least in part) to technical artifacts, resulting in a higher degree of artificial duplication in the assembly of its genome, as suggested by the higher BUSCO score obtained for the "complete \& duplicated" hits (Supplementary Fig. S4). Nonetheless, the possibility that L.d. japonica's genome does feature a greater number of genes than that of L.d. asiatica should not be overlooked. A complementary reciprocal best hit $(\mathrm{RBH})$ analysis performed during post-processing steps further revealed that 10,440 genes possess nearly identical protein sequences between L.d. asiatica and L.d. japonica, of which $99.6 \%$ were annotated with a putative gene product (Fig. 2a, Supplementary Table S4). This additional layer of information adds to the existing gene product, gene ontology and KEGG functional pathway assignment. It can be used as an extra measure of confidence that a given gene is not a false positive because it has been characterized in multiple complementary analyses. An additional orthologous analysis comparing whole genome sequences of 12 lepidopteran species with the AGM genomes sequenced here corroborated previous phylogenetic relationships among these taxa ${ }^{22-24}$ (Fig. 2b, Supplementary information S3). The analysis also confirmed the highly repeated nature of AGM genomes, showing numerous many-to-one orthologous relationships with other lepidopteran taxa, which explains some of the low bootstrap values obtained in the final rooted species tree generated by orthoFinder (Fig. $2 \mathrm{~b}$ ). These results suggest that despite the presence of numerous repetitive sequences, gene models generated for each of the two AGM subspecies remain of high quality. Complimentary gapped BLASTn analyses of the published EGM transcriptome ${ }^{10}$ against our AGM genomes revealed that $98 \%$ and $99 \%$ of the transcripts align onto the genome of L.d. asiatica and L.d. japonica, respectively (with an average $99 \%$ identities for both genomes). Overall, the present results indicate that the genomes generated in this study can be used as reliable draft reference assemblies. Future genome versions will incorporate data from complementary sequencing platforms to increase N50s and polish these reference sequences.

High prevalence of transposable elements in L. dispar spp. genomes. The AGM genomes assembled contained large proportions of repetitive DNA spread across all contigs, most of which are transposable elements (TEs) described in other animal species. Nearly $60 \%$ of the genomes of L.d asiatica and L.d. japonica consist of TEs and repeated sequences. Long Interspersed Nuclear Elements (LINEs) are the most abundant TEs identified in both genomes, accounting for $28 \%$ and $26 \%$ of all genomic DNA (gDNA) in L.d. asiatica and L.d. japonica, respectively (Fig. 3a). Interestingly, the second most abundant group of repeated elements ( $12 \%$ and $13 \%$ of all gDNA in L.d. asiatica and L.d. japonica, respectively) were novel, "unclassified" elements that were identified through the creation of a de novo repeat library (Supplementary Information Section S2). 


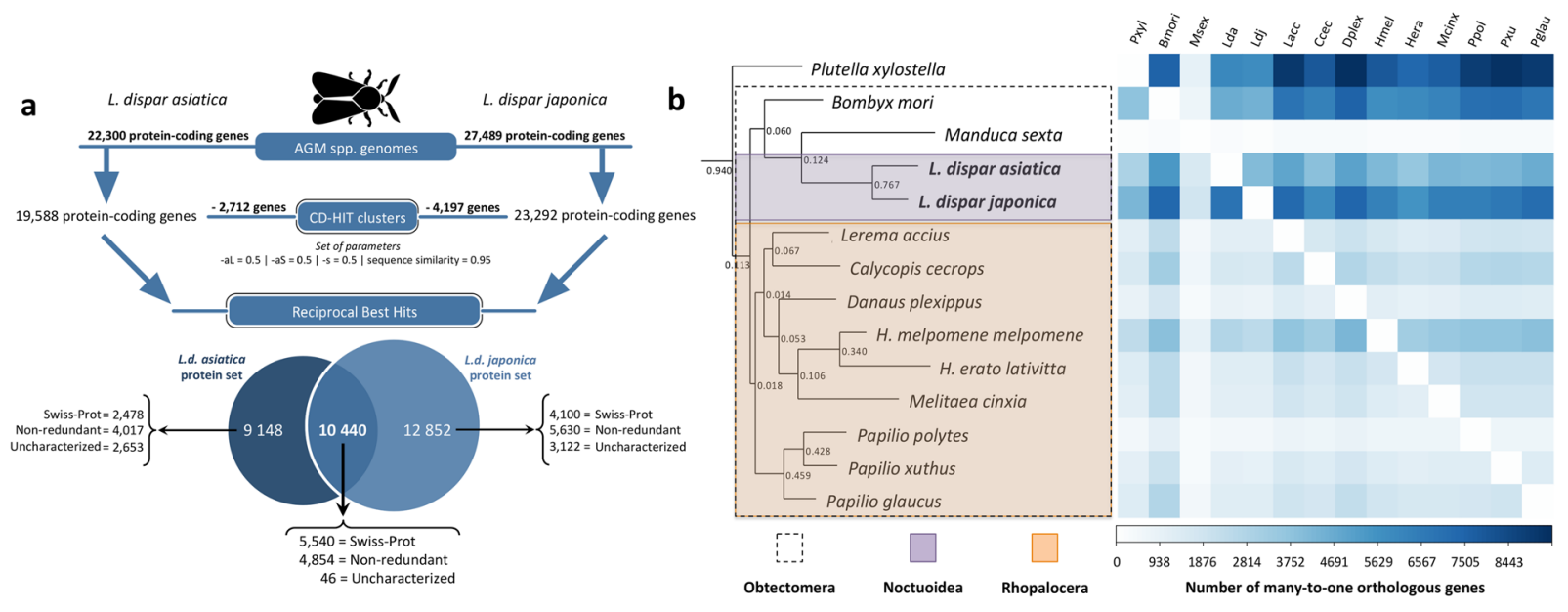

Figure 2. Genome assembly post-processing steps. (a) Gene models were refined by (i) reducing redundancy using the program CD-HIT and (ii) assessing protein sequence similarities between L.d. asiatica and L.d. japonica using a reciprocal best hit approach with BLASTp. (b) Rooted species tree inferred from gene trees generated by orthoFinder for all amino acid orthogroups shared among 14 Lepidopteran taxa (including AGM) encompassing the Noctuoidea (orange) and Rhopalocera (violet) superfamilies of the Obtectomera clade (dashed box). The heatmap on the right represents pairwise comparisons between all species included in the analysis, showing the number of many-to-one $(\mathrm{N}: 1)$ orthologous genes between each species pair.
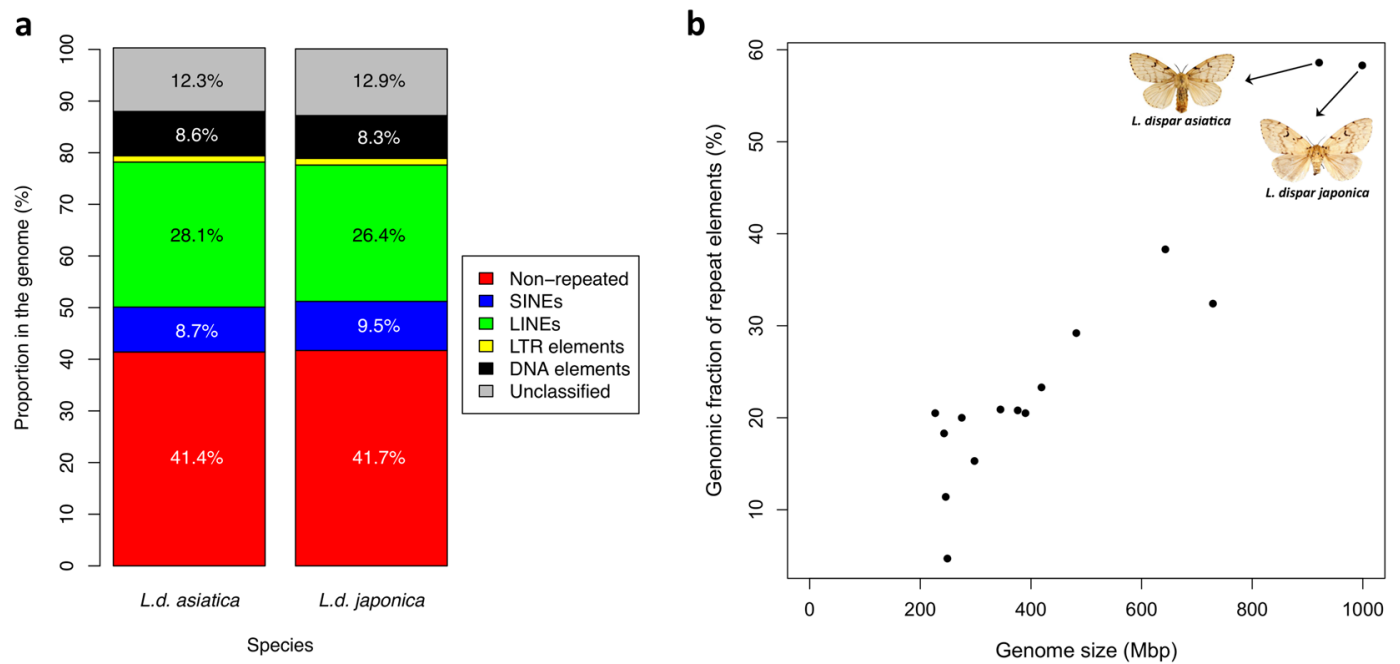

Figure 3. High content of repeated sequences in AGM. (a) Proportion of specific repeated element categories in L.d. asiatica (black) and L.d. japonica (white) genome assemblies. (b) Total percentage of repeat elements in the genome is positively correlated with genome size across Lepidopteran species. Data on genome sizes and repeat elements from 13 Lepidopteran species (Bombyx mori, Calycopis cecrops, Danaus plexippus, Heliconius melpomene, Leptidea sinapis, Lerema accius, Manduca sexta, Melitaea cinxia, Papilio glaucus, Papilio polytes, Papilio xuthus, Papilio sennae, Papilio rapae) were taken from Talla et al. ${ }^{20}$ (see Supplementary Table S3). L.d. asiatica photograph taken by Alexander Schintlmeister and L.d. japonica photograph taken by Ken Walker for the Museum Victoria, PaDIL (CC BY $3.0 \mathrm{au}$ ).

Short Interspersed Nuclear Elements (SINEs) are the third most abundant TEs, comprising $10 \%$ and $9 \%$ of L.d. asiatica and L.d. japonica genomes, respectively. Both AGM genomes exhibit considerable difference in total proportion of TEs and occurrence of certain TE classes when compared to most other Lepidopteran taxa. The largest proportion of TEs observed in a lepidopteran genome before this study was in Leptidea sinapis (38.3\%), while other taxa exhibit total TE proportions between $4.7 \%$ and $29.2 \%{ }^{20}$. The proportion of LINEs in the two AGM assemblies are particularly high compared to other lepidopteran species, for which estimates vary from $0.7 \%$ (common Mormon, Papilio polytes) to $5.4 \%$ (white-wood butterfly, Leptidea sinapis) ${ }^{20}$. The results presented here for L.d. asiatica and L.d. japonica support and extend a strong correlation (Pearson's; $\mathrm{n}=15, \mathrm{R}=0.95, \mathrm{r}^{2}=0.908$, $\mathrm{P}$-value $=4.25 \times 10^{-8}$ ) between genome size and repeat content in lepidopteran genomes (Fig. 3b, Supplementary 


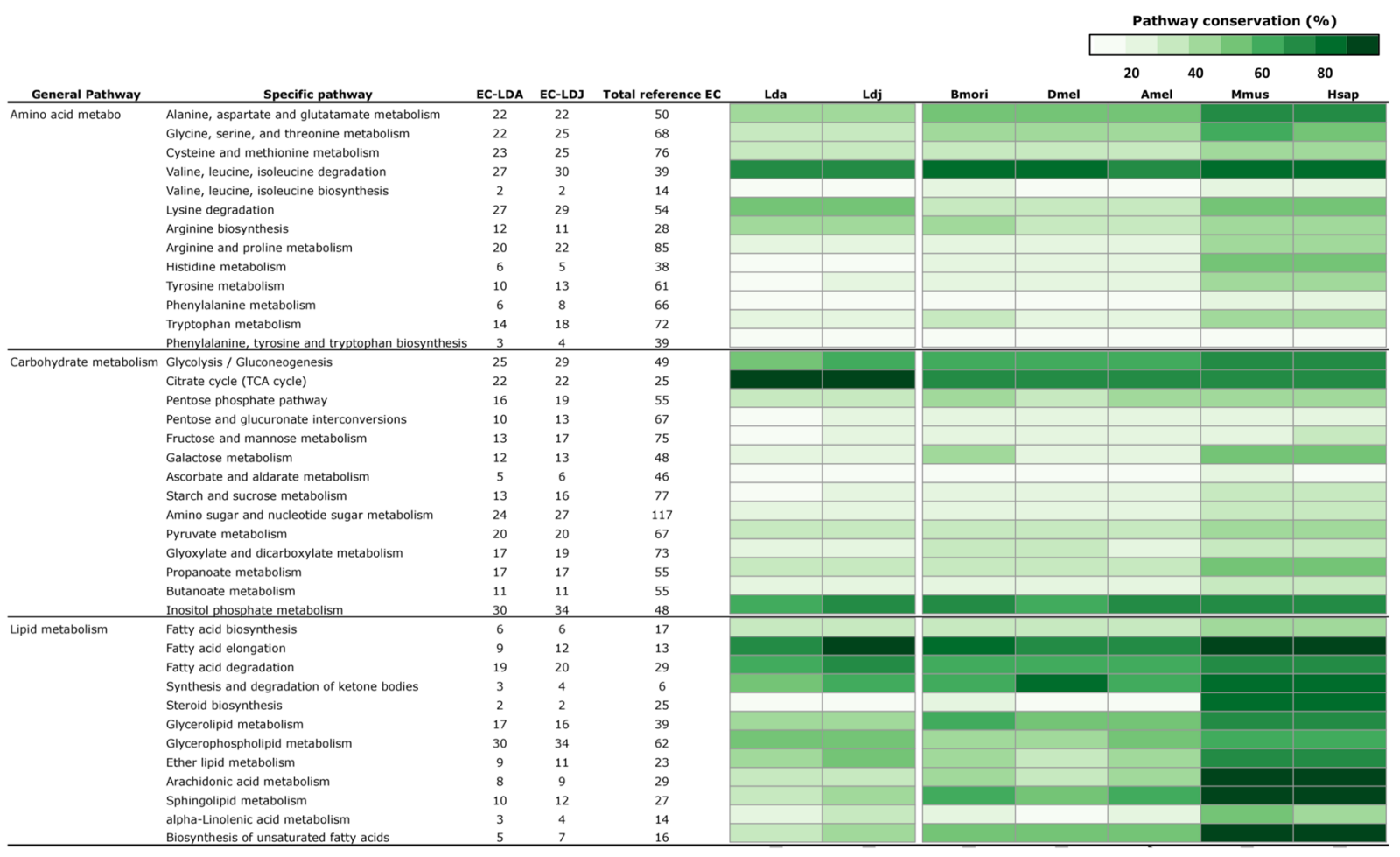

Figure 4. Conservation of genome-wide KEGG metabolic pathways. Characterization and comparisons of metabolic pathways across three different insect orders (Bombyx mori - Lepidoptera, Apis mellifera Hymenoptera, Drosophila melanogaster - Diptera) and two well-characterized mammalian species (Mus musculus and Homo sapiens) in comparison with L.d. asiatica and L.d. japonica (Lepidoptera). Rows represent specific metabolic pathways grouped in three general categories, i.e. amino acid metabolism, carbohydrate metabolism, and lipid metabolism, as defined by the Kyoto Encyclopedia of Genes and Genomes (KEGG). Colors represent the species-specific pathway conservation level, defined as the percentage of enzymes identified in the genome as compared to the KEGG reference pathway. Amel $=$ Apis mellifera, Bmori $=$ Bombyx mori, Dmel $=$ Drosophila melanogaster, $\mathrm{Lda}=$ Lymantria dispar asiatica, $\mathrm{Ldj}=$ Lymantria dispar japonica, Hsap $=$ Homo sapiens.

Table S2). This relationship has also been reported for insects ${ }^{25}$, fishes ${ }^{26,27}$, flowering plants ${ }^{28}$, birds and mammals ${ }^{29}$, and trees ${ }^{30,31}$.

The pronounced expansion of LINEs and unclassified elements in AGM genomes supports the idea that TE activity plays a strong role in genome size evolution in the Lepidoptera, and potentially in eukaryotes in general. This could have significant functional implications in terms of gene regulatory networks, as TE-derived sequences may be co-opted for cis-regulatory elements in the genome ${ }^{32-35}$. Recent research on both prokaryotic and eukaryotic invasive species suggests that interactions between environmental stresses and activity of transposable elements could fuel rapid adaptation to new environments and promote invasiveness through genomic structural changes or other innovations ${ }^{36-39}$. Characterizing TEs diversity and identifying their precise genomic locations in AGM could also help develop a foundation for exploring gypsy moth gene regulation.

Reconstruction of metabolic pathways for different insect species. Metabolic pathway reconstruction analysis showed a relatively high conservation level in most pathways across various taxa, with small differences in several pathways in AGM (Fig. 4). A recent large-scale metabolic investigation in AGM, based on transcriptomics, revealed a significantly greater number of unique transcripts associated with chitin degradation than chitin biosynthesis, two crucial processes taking place during growth and development ${ }^{12}$. We confirmed this trend, identifying 32 genes involved in chitin catabolism (mostly chitinases) and 16 genes involved in chitin biosynthetic pathways (Supplementary Tables S5 and S6). Considering that chitin is the major biochemical component of insect cuticle that must be produced and degraded rapidly during molting phases at the larval stage (the developmental stage at which intense forest defoliation occurs during AGM outbreaks), characterizing genes involved in chitin-related metabolism offers promising molecular targets from which new species-specific biocontrol strategies could be developed ${ }^{40}$ (e.g., blocking chitin metabolism to prevent growth and development). Another aspect of chitin-related genes that may have major implications for AGM invasiveness is cold tolerance. Transcriptomics results obtained from eight species of stick insects ${ }^{41,42}$ and in the seabuckthorn carpenter moth (Eogystia hippophaecolus, see ${ }^{43}$ ) suggested a parallel pathway to cold tolerance adaptation based on cuticle-related genes. The three gene products that systematically responded with high levels of expression at low temperature $^{41}$ (prolyl 4-hydroxylase subunit alpha-1, staphylococcal nuclease domaincontaining protein 1 and a cuticular 
protein gene), along with 90 and 103 cuticle-related gene products, have been fully annotated in the present L.d. asiatica and L.d. japonica genome assemblies, respectively (Supplementary Tables S5 and S6).

Specific pathways related to chitin metabolism are part of a more global "carbohydrate metabolism" pathway, which also features the most conserved pathway identified in AGM subspecies: the citrate cycle (TCA cycle or Krebs cycle, Fig. 4). This citrate cycle performs the last steps of the oxidation of carbohydrates and fatty acids. In total, 22 enzymes belonging to this pathway, out of the 25 enzymes that compose the full KEGG reference pathway, were identified in the genomes of L.d. asiatica and L.d. japonica. In comparison to the other taxa included in the analysis, this result indicates that AGM genomes contain diversified enzymatic tools associated with several biochemical pathways regulating energy production.

Thermogenesis is another energy-related metabolic pathway that we explored and that could play a significant role in the determination of flight capacity and cold tolerance in AGM (KEGG pathway number ko04714). This pathway came second in number of successful hits on the KEGG database. In total, 102 and 114 unique KEGG numbers were identified in the genomes of L.d. asiatica and L.d. japonica, respectively. These were associated with 19 (L.d. asiatica) and 23 (L.d. japonica) unique EC enzymes involved in thermogenesis (Supplementary Table S7). This result suggests that in AGM, the importance of energy metabolism may also be closely linked with its flight capability, which requires high fluxes of energy executed at high core metabolic temperatures ${ }^{44,45}$. Moths are recognized for their high capacity for evasiveness during flight, having the ability to quickly initiate flight, attain varying speeds and change direction rapidly and erratically ${ }^{46}$. Moths are also heterothermic, which means they cannot perform these energy-consuming activities efficiently at low ambient temperatures ${ }^{47}$. As a result, AGM likely need to control their body temperature with high precision through various regulatory mechanisms in order to fly ${ }^{48}$. In insects, thermogenesis is activated during multiple biological activities, such as flying, running, singing, pre-flight warm-up, and social activities ${ }^{49}$. In this context, thermogenesis and locomotive functions are closely connected. Disentangling these issues will require specific hypothesis-driven functional experiments, and the genome assemblies reported here provide a key resource to meet that challenge. AGM genomes exhibit a broad metabolic competence by encoding a wide variety of enzymes required to perform flight, feed on multiple different hosts, and tolerate cold temperatures, traits strongly implicated in the invasive nature of these insects.

Genome-wide characterization of developmental genes. One core question pertaining to the ecological differences between AGM subspecies is how and why traits associated with flight capability vary in different Lymantria dispar ssp. populations. Data on wing morphometrics from eight geographic populations of Lymantria dispar spp., including EGM and AGM subspecies, has shown that females from populations with strong directed flight capabilities have significantly lower wing load (i.e. body mass divided by total wing area) than females from flightless populations ${ }^{50}$. These phenotypic differences, along with differences in population-specific flight muscle capacities, can predict flight capabilities in individuals from unknown source populations. Since flight-related muscle function is strongly correlated with the flightless phenotype, we assessed the possible presence of a loss-of-function mutations within the coding sequence of flightin $(f \mathrm{ln})$, a gene required for proper direct flight muscles assembly in Drosophila melanogaster ${ }^{51}$. In other species, coding sequence mutations have been implicated in sexually dimorphic trait expression when regulated by modifiers or dosage compensation ${ }^{52,53}$. However, we found no evidence for $f l n$ coding sequence evolution underlying flight capability differences between EGM and AGM (see Supplemental Information, Section S5 for further details).

We also characterized the presence and structure of homeotic genes in the AGM genomes (summarized in Supplementary Fig. S5), in order to test the possibility that differences in female flight capability are the result of different developmental trajectories between flight capable and flight incapable individuals. Homeotic genes encode crucial transcription factors involved in body patterning and developmental trajectories ${ }^{54}$. These genes could harbor intrinsic sequence differences or gene copy numbers among AGM subspecies that could be involved in different developmental trajectories, resulting in different wing loads or varying muscle strength capacities. We identified a collection of 87 (L.d. asiatica) and 111 (L.d. japonica) homeotic genes, spanning a total of 60 unique homeobox gene families and distributed across ten different gene classes. No major structural rearrangements (e.g. cluster organization, expansion/retraction of certain families) were detected between homeotic genes identified in AGM, as compared to other Lepidopteran species (see Supplementary Information Section S4 for further details). Our description of AGM hox and other homeotic genes however supplies key resources for exploring other potential developmental causes to differential flight capacity (e.g. population-specific SNPs in key developmental genes), and appendage formation in gypsy moths and the Lepidoptera in general.

\section{Conclusion}

Although AGM moths are not currently established in North America, they represent a constant threat to global forest ecosystem stability due to their strong invasive capacity. The novel reference genomes presented in this study provide valuable genomic information that can be used to better understand the biological characteristics that make AGM one of the top threatening forest pests in North America. This study also shows that it is possible to assemble a large and complex genome with a single long read dataset, in this case PacBio. It shows that smaller research teams with limited resources can build reliable reference genomes that match most of the high-quality genomic references obtained with more elaborate datasets. Moths and butterflies show variable genome sizes and complexities. Our genome assembly pipeline, specifically developed to recognize lepidopteran genes, revealed that AGM possess the largest genomes sequenced to date among Lepidoptera, a characteristic that can be explained by the accumulation of species-specific transposable elements. Large-scale genomic data generated in this study will help in the identification of the genetic basis of key traits defining the invasive capacity of AGM, such as host diet breadth, cold tolerance, and female flight capacity. Population-scale investigations based on the reference assemblies generated here will also enable the development of highly specific molecular diagnostic tools to create fine-tuned monitoring and managing strategies for future AGM outbreaks. 


\begin{abstract}
Methods
Sequencing, genome assembly and annotation. Genome sequencing was carried out using the Single Molecule, Real-Time technology (SMRT) developed by Pacific Biosciences (PacBio). We developed an 8-step custom-made genome assembly and annotation pipeline available on Github (https:/github.com/fohebert/ GenomeAnnotation, see Supplementary Information Section S2 for further details). Complete raw sequence data obtained from four different specimens (Supplementary Information Section S2) was registered under the NCBI BioProject IDs PRJNA479680 (L.d. asiatica) and PRJNA479831 (L.d. japonica), associated with NCBI Sequence Read Archive (SRA) accession numbers SAMN09601828 (L.d. asiatica) and SAMN09601829 (L.d. japonica). Steps in the pipeline include (1) the assembly of raw sequencing reads into longer contig sequences using Canu v.1.5 $\left(\right.$ genomeSize $=1.0 \mathrm{~g}$, maxMemory $=800, \max T h r e a d s=60$, all other parameters kept to default $\operatorname{mode}{ }^{55}$ ). (2) Identification and masking of repeated DNA. A Lymantria-specific repetitive DNA database was first created using RepeatModeler v.1.0.8 ${ }^{56}$ ('-engine ncbi', all other parameters set at default values) and was then used in combination with the curated Repbase library of repeats ${ }^{57}$ to screen the AGM genomes for specific classes of repeats using RepeatMasker v.4.0.6 ${ }^{58}$. (3) Identification of protein-coding genes in AGM genomic sequences through pairwise sequence comparison. This step was conducted through two complementary approaches implemented in the programs Exonerate v.2.4.0 $0^{59}$ and SciPio v.1.4.1 ${ }^{60}$. (4) Lymantria-specific mRNA transcript alignments onto each of the two AGM genomes using PASA v.2.1.061. (5) Genome-wide ab initio identification of gene sequences using the program GeneMark-ES v.1.062. (6) Computation of weighted consensus gene structure annotations based on the evidence gathered through steps three to five, using EVidenceModeler (EVM) v.1.1.1 ${ }^{63}$. (7) Genomewide identification of protein-coding genes by training AUGUSTUS v.3.2.2 ${ }^{64}$ on a high-quality gene set identified through EVM in the previous step, and then running the trained version of the program on the complete AGM genomes. (8) Assignment of gene products and Gene Ontology (GO) terms to AGM protein-coding genes using BLASTp searches in NCBI's UniprotKB/Swiss-Prot and non-redundant protein databases. Post-processing steps were also performed to polish final contig sequences using PacBio's GenomicConsensus package (https://github. com/PacificBiosciences/GenomicConsensus) and eliminate redundancy in gene models (see Supplementary Information Section S3 for further details). We ultimately clustered similar amino acid sequences into consensus gene models using CD-HIT ${ }^{65}$ and performed whole-genome sequence comparisons with 12 other Lepidopteran species to identify orthology relationships, using orthoFinder ${ }^{66}$ (Supplementary Information Section S3).
\end{abstract}

Metabolic pathway reconstruction. We assigned KO (KEGG Orthology) numbers to the amino acid sequences obtained through our assembly pipeline using the BlastKOALA annotation server ${ }^{67}$, which allowed the reconstruction of various molecular networks such as carbohydrate, lipid and amino acid enzymatic pathways. To perform this analysis, we retrieved the Enzyme Nomenclature (EC) numbers based on the KO numbers (ECs are attributes of KEGG Orthology numbers) in the raw BlastKOALA output, and estimated the percentage of conservation for each specific pathway, defined as the percentage of enzymes identified in a species-specific pathway as compared to the KEGG reference pathway. We compared the results to five other species: Bombyx mori (silk worm); Drosophila melanogaster (fruitfly); Apis mellifera (European honey bee); Mus musculus (mouse), and; Homo sapiens (human).

Identification and phylogeny of homeotic genes. Complete sets of homeotic genes from 10 different animal species were downloaded from the HomeoDB ${ }^{2}$ website ${ }^{68}$ : human (Homo sapiens); mouse (Mus musculus); chicken (Gallus gallus); frog (Xenopus [Silurana] tropicalis); zebrafish (Danio rerio); amphioxus (Branchiostoma floridae); fruitfly (Drosophila melanogaster); red flour beetle (Tribolium castaneum); western honeybee (Apis mellifera), and; nematode (Caenorhabditis elegans). We used these gene sequences to build a local database to which all of the amino acid sequences predicted from the genomes of L.d. asiatica and L.d. japonica were compared, using the sequence similarity algorithm implemented in NCBI's BLASTp program v.2.6.0 ${ }^{69}$. BLASTp results exhibiting an e-value $<1$ e- 15 and an overall identity value $>40 \%$ were considered significant and were kept for downstream phylogenetic analysis. All significant BLASTp results were further manually validated using the Simple Modular Architecture Research Tool (SMART) ${ }^{70,71}$, in combination with a visual assessment of the BLASTp result to confirm that the candidate homeotic genes truly contain at least one homeodomain sequence. Pseudogenes were annotated but discarded from downstream analyses. Manually validated homeodomain sequences resulting from the sequence homology analysis were aligned using CLUSTAL-OMEGA v.1.2.4 ${ }^{72}$ with mBed-like clustering guide-tree and iteration, a maximum of five combined iterations, five guide tree iterations, and five Hidden Markov Model (HMM) iterations (all other parameters set at default values). The resulting alignment was used to construct a phylogenetic tree with RAxML v.8.2.0 $0^{73}$, following a gamma model rate of heterogeneity, combined with a WAG substitution matrix and a maximum likelihood search of 100 bootstraps. Final trees were generated and edited using FigTree v.1.4.3 (http://tree.bio.ed.ac.uk/software/figtree/).

\title{
Data availability
}

The raw sequencing files used to generate the genome assemblies analyzed during the current study were registered as NCBI BioProject IDs PRJNA479680 (L.d. asiatica) and PRJNA479831 (L.d. japonica), associated with NCBI Sequence Read Archive (SRA) accession numbers SAMN09601828 (L.d. asiatica) and SAMN09601829 (L.d. japonica).

The genome assembly pipeline used to analyze the raw datasets, with corresponding Perl, Python and bash utility scripts, as well as final FASTA/GFF3 genome files, are available on Github: https://github.com/fohebert/ GenomeAnnotation, as well as on the Open Science Framework public repository: https://doi.org/10.17605/OSF. IO/UNZ2V.

All other data generated/analyzed during this study are otherwise included in this published article and its Supplementary Files. 
Received: 30 January 2019; Accepted: 18 October 2019;

Published online: 11 November 2019

\section{References}

1. Pogue, M. G. \& Schaefer, P. W. A review of selected species of Lymantria Hübner (1819) including three new species (Lepidoptera: Noctuidae: Lymantriinae) from subtropical and temperate regions of Asia, some potentially invasive to North America. U.S. Dept. of Agriculture, Forest Health Technology Enterprise Team (Washington, D.C., 2007).

2. Dumouche, L. Plant health risk assessment: Asian gypsy moth. Canadian Food Inspection Agency (2010).

3. USDA. Pest alert - Asian gypsy moth. APHIS 81-35-027 leaflet. United States Department of Agriculture (2016).

4. Baranchikov, Y. N. \& Sukachev, V. N. In Proceedings, Lymantriidae a comparison of features of New and Old World tussock moths (eds Wallner, W. E. \& McManus, K. A.) (1989).

5. Wallner, W. E., Humble, L. M., Levin, R. E., Baranchikov, Y. N. \& Carde, R. T. Response of adult lymantriid moths to illumination devices in the Russian Far East. Journal of Economic Entomology 88, 337-342 (1995).

6. Keena, M. A. Comparison of the hatch of Lymantria dispar (Lepidoptera: Lymantriidae) eggs from Russia and the United States after exposure to different temperatures and durations of low temperature. Annals of the Entomological Society of America 89, 564-572 (1996).

7. Keena, M. A. Inheritance and world variation in thermal requirements for egg hatch in Lymantria dispar (Lepidoptera: Erebidae). Environ. Entomol. 45, 1-10 (2016).

8. Keena, M. A., Côté, M.-J., Grinberg, P. S. \& Wallner, W. E. World distribution of female flight and genetic variation in Lymantria dispar (Lepidoptera: Lymantriidae). Environ. Entomol. 37, 636-649 (2008).

9. Keena, M. A., Wallner, W. E., Grinberg, P. S. \& Carde, R. T. Female Flight Propensity and Capability in Lymantria dispar (Lepidoptera: Lymantriidae) from Russia, North America, and Their Reciprocal F. Annals of the Entomological Society of America 30, 380-387 (2001)

10. Sparks, M. E., Blackburn, M. B., Kuhar, D. \& Gundersen-Rindal, D. E. Transcriptome of the Lymantria dispar (Gypsy Moth) Larval Midgut in Response to Infection by Bacillus thuringiensis. Plos One 8, e61190-9 (2013).

11. Cao, C. et al. Characterization of the transcriptome of the Asian gypsy moth Lymantria dispar identifies numerous transcripts associated with insecticide resistance. Pesticide Biochemistry and Physiology 119, 54-61 (2015).

12. Xiaojun, F., Chun, Y., Jianhong, L., Chang, Z. \& Yao, L. Sequencing and de novo assembly of the Asian gypsy moth transcriptome using the Illumina platform. Genet. Mol. Biol. 40, 160-167 (2017).

13. Zhang, J. et al. Gypsy moth genome provides insights into flight capability and virus-host interactions. P Natl Acad Sci Usa 13, 201818283-10 (2019).

14. Petitpierre, E. Molecular cytogenetics and taxonomy of insects, with particular reference to the Coleoptera. International Journal of Insect Morphology and Embryology 25, 115-133 (1996).

15. Simão, F. A., Waterhouse, R. M., Ioannidis, P. \& Zdobnov, E. BUSCO: assessing genome assembly and annotation completeness with single-copy orthologs. Bioinformatics, https://doi.org/10.1093/bioinformatics/btv351/-/DC1 (2015).

16. Gschloessl, B. et al. Draft genome and reference transcriptomic resources for the urticating pine defoliator Thaumetopoea pityocampa (Lepidoptera: Notodontidae). Mol Ecol Resour 18, 602-619 (2018).

17. International Silkworm Genome Consortium. The genome of a lepidopteran model insect, the silkworm Bombyx mori. Insect Biochem. Mol. Biol. 38, 1036-1045 (2008).

18. Lange, A. et al. Genome Sequence of Galleria mellonella(Greater Wax Moth). Genome Announc 6, e01220-17-2 (2018).

19. Kim, S.-R. et al. Genome sequence of the Japanese oak silk moth, Antheraea yamamai: the first draft genome in the family Saturniidae. Gigascience 7, 1-11 (2018).

20. Talla, V. et al. Rapid increase in genome size as a consequence of transposable element hyperactivity in wood-white (Leptidea) butterflies. Genome Biol. Evol. 9, 2491-2505 (2017).

21. Challis, R. J., Kumar, S., Dasmahapatra, K. K. K., Jiggins, C. D. \& Blaxter, M. Lepbase: the Lepidopteran genome database. bioRxiv 1-15, https://doi.org/10.1101/056994 (2016).

22. Cong, Q., Borek, D., Otwinowski, Z. \& Grishin, N. V. Skipper genome sheds light on unique phenotypic traits and phylogeny. BMC Genomics 16, 1-13 (2015)

23. Cong, Q., Borek, D., Otwinowski, Z. \& Grishin, N. V. Tiger swallowtail genome reveals mechanisms for speciation and caterpillar chemical defense. Cell Reports 10, 910-919 (2015).

24. Cong, Q. et al. Complete genomes of Hairstreak butterflies, their speciation, and nucleo-mitochondrial incongruence. Sci. Rep. 6, $1-15$ (2016).

25. Wang, X. et al. The locust genome provides insight into swarm formation and long-distance flight. Nature Communications 5, 2957 (2014).

26. Aparicio, S. et al. Whole-genome shotgun assembly and analysis of the genome of Fugu rubripes. Science 297, 1301-1310 (2002).

27. Blass, E., Bell, M. \& Boissinot, S. Accumulation and rapid decay of non-LTR retrotransposons in the genome of the three-spine stickleback. Genome Biol. Evol. 4, 687-702 (2012).

28. Bennetzen, J. L., Ma, J. \& Devos, K. M. Mechanisms of recent genome size variation in flowering plants. Ann. Bot. 95, 127-132 (2005).

29. Kapusta, A., Suh, A. \& Feschotte, C. Dynamics of genome size evolution in birds and mammals. Proceedings of the National Academy of Sciences 114, E1460-E1469 (2017).

30. Vicient, C. et al. Retrotransposon BARE-1 and its role in genome evolution in the genus Hordeum. Plant Cell 11, 1769-1784 (1999).

31. Nystedt, B. et al. The Norway spruce genome sequence and conifer genome evolution. Nature Publishing Group 497, 579-584 (2013).

32. Slotkin, R. K. \& Martienssen, R. Transposable elements and the epigenetic regulation of the genome. Nat Rev Genet 8, 272-285 (2007).

33. Feschotte, C. Transposable elements and the evolution of regulatory networks. Nat Rev Genet 9, 397-405 (2008).

34. Chuong, E. B., Elde, N. C. \& Feschotte, C. Regulatory activities of transposable elements: from conflicts to benefits. Nat Rev Genet 18, 71-86 (2017).

35. Trizzino, M. et al. Transposable elements are the primary source of novelty in primate gene regulation. Genome Res $27,1623-1633$ (2017).

36. Stapley, J., Santure, A. W. \& Dennis, S. R. Transposable elements as agents of rapid adaptation may explain the genetic paradox of invasive species. Molecular Ecology 24, 2241-2252 (2015).

37. Dennenmoser, S. et al. Copy number increases of transposable elements and protein-coding genes in an invasive fish of hybrid origin. Molecular Ecology 26, 4712-4724 (2017).

38. Gillings, M. R. Class 1 integrons as invasive species. Curr. Opin. Microbiol. 38, 10-15 (2017).

39. Dale, A. L. et al. Mitotic recombination and rapid genome evolution in the invasive forest pathogen phytophthora ramorum. MBio 10, 81 (2019).

40. Nagpure, A., Choudhary, B. \& Gupta, R. K. Chitinases: in agriculture and human healthcare. Critical Reviews in Biotechnology 34, 215-232 (2013). 
41. Dunning, L. T. et al. Identification of cold-responsive genes in a New Zealand alpine stick insect using RNA-Seq. Comparative Biochemistry and Physiology - Part D: Genomics and Proteomics 8, 24-31 (2013).

42. Dunning, L. T., Dennis, A. B., Sinclair, B. J., Newcomb, R. D. \& Buckley, T. R. Divergent transcriptional responses to low temperature among populations of alpine and lowland species of New Zealand stick insects (Micrarchus). Molecular Ecology 23, 2712-2726 (2014).

43. Cui, M., Hu, P., Wang, T., Tao, J. \& Zong, S. Differential transcriptome analysis reveals genes related to cold tolerance in seabuckthorn carpenter moth, Eogystia hippophaecolus. Plos One 12, e0187105-16 (2017).

44. Withers, P. C. The effects of ambient air pressure on oxygen consumption of resting and hovering honeybees. J Comp Physiol B 141, 433-437 (1981)

45. Yang, Y., Xu, S., Xu, J., Guo, Y. \& Yang, G. Adaptive evolution of mitochondrial energy metabolism genes associated with increased energy demand in flying insects. Plos One 9, e99120 (2014).

46. Jantzen, B. \& Eisner, T. Hindwings are unnecessary for flight but essential for execution of normal evasive flight in Lepidoptera. Proceedings of the National Academy of Sciences 105, 16636-16640 (2008).

47. Iwaizumi, R., Arakawa, K. \& Koshio, C. Nocturnal flight activities of the female Asian gypsy moth, Lymantria dispar (Linnaeus) (Lepidoptera: Lymantriidae). Appl. Entomol. Zool. 45, 121-128 (2010).

48. Loli, D. \& Bicudo, J. E. P. W. Control and regulatory mechanisms associated with thermogenesis in flying insects and birds. Biosci. Rep. 25, 149-180 (2005).

49. Block, B. A. Thermogenesis in muscle. Annu. Rev. Physiol. 56, 535-577 (1994).

50. Shi, J., Chen, F. \& Keena, M. A. Differences in wing morphometrics of Lymantria dispar (Lepidoptera: Erebidae) between populations that vary in female flight capability. Annals of the Entomological Society of America 108, 528-535 (2015).

51. Contompasis, J. L., Nyland, L. R., Maughan, D. W. \& Vigoreaux, J. O. Flightin is necessary for length determination, structural integrity, and large bending stiffness of insect flight muscle thick filaments. J Mol Biol 395, 340-348 (2010).

52. Williams, T. M. \& Carroll, S. B. Genetic and molecular insights into the development and evolution of sexual dimorphism. Nat Rev Genet 10, 797-804 (2009)

53. Parsch, J. \& Ellegren, H. The evolutionary causes and consequences of sex-biased gene expression. Nat Rev Genet 14, 83-87 (2013).

54. Holland, P. W. H. Evolution of homeobox genes. WIREs Dev Biol 2, 31-45 (2012).

55. Koren, S. et al. Canu: scalable and accurate long-read assembly via adaptive k-mer weighting and repeat separation. Genome Res 27, 722-736 (2017).

56. Smit, A. F. \& Hubley, R. RepeatModeler Open-1.0. (2008-2015), http://www.repeatmasker.org.

57. Bao, W., Kojima, K. K. \& Kohany, O. Repbase Update, a database of repetitive elements in eukaryotic genomes. Mobile DNA 1-6, https://doi.org/10.1186/s13100-015-0041-9 (2015).

58. Smit, A. F., Hubley, R. \& Green, P. RepeatMasker Open-4.0. (2013-2015), http://www.repeatmasker.org.

59. Slater, G. S. C. \& Birney, E. Automated generation of heuristics for biological sequence comparison. BMC Bioinformatics 6, 31 (2005).

60. Keller, O., Odronitz, F., Stanke, M., Kollmar, M. \& Waack, S. Scipio: Using protein sequences to determine the precise exon/intron structures of genes and their orthologs in closely related species. BMC Bioinformatics 9, 278-12 (2008).

61. Haas, B. J. et al. Improving the Arabidopsis genome annotation using maximal transcript alignment assemblies. Nucleic Acids Res 31, 5654-5666 (2003).

62. Lomsadze, A., Ter-Hovhannisyan, V., Chernoff, Y. O. \& Borodovsky, M. Gene identification in novel eukaryotic genomes by selftraining algorithm. Nucleic Acids Res 33, 6494-6506 (2005).

63. Haas, B. J. et al. Automated eukaryotic gene structure annotation using EVidenceModeler and the Program to Assemble Spliced Alignments. Genome Biol 9, R7 (2008)

64. Stanke, M., Schöffmann, O., Morgenstern, B. \& Waack, S. Gene prediction in eukaryotes with a generalized hidden Markov model that uses hints from external sources. BMC Bioinformatics 7, 62 (2006).

65. Huang, Y., Niu, B., Gao, Y., Fu, L. \& Li, W. CD-HIT Suite: a web server for clustering and comparing biological sequences. Bioinformatics 26, 680-682 (2010).

66. Emms, D. M. \& Kelly, S. OrthoFinder: solving fundamental biases in whole genome comparisons dramatically improves orthogroup inference accuracy. Genome Biol 16, 1-14 (2015).

67. Kanehisa, M., Sato, Y. \& Morishima, K. BlastKOALA and GhostKOALA: KEGG Tools for Functional Characterization of Genome and Metagenome Sequences. J Mol Biol 428, 726-731 (2016).

68. Zhong, Y.-F. \& Holland, P. W. H. HomeoDB2: functional expansion of a comparative homeobox gene database for evolutionary developmental biology. Evol Dev 13, 567-568 (2011).

69. Camacho, C. et al. BLAST+: architecture and applications. BMC Bioinformatics 10, 1 (2009).

70. Schultz, J., Milpetz, F., Bork, P. \& Ponting, C. P. SMART, a simple modular architecture research tool: identification of signaling domains. P Natl Acad Sci Usa 95, 5857-5864 (1998).

71. Letunic, I. \& Bork, P. 20 years of the SMART protein domain annotation resource. Nucleic Acids Res 46, D493-D496 (2017).

72. Sievers, F. et al. Fast, scalable generation of high-quality protein multiple sequence alignments using Clustal Omega. Mol. Syst. Biol. 7, 1-6 (2011)

73. Stamatakis, A. RAxML version 8: a tool for phylogenetic analysis and post-analysis of large phylogenies. Bioinformatics 30, $1312-1313(2014)$.

\section{Acknowledgements}

We would like to thank Drs. Hannah Nade and Melody Keena (USDA-APHIS) for the rearing and provision, respectively, of adult specimens of Lymantria dispar asiatica and Lymantria dispar japonica. This work was funded by Genome Canada, Genome British Columbia, and Genome Quebec, through a Large Scale Applied Research Project Competition grant.

\section{Author contributions}

F.O.H., L.F., B.B., M.C., K.D., R.C.H. and R.C.L. designed the work; F.O.H. performed the assembly and downstream bioinformatics/statistical analyses and drafted the manuscript; L.F. helped acquire a first draft assembly of the L. dispar asiatica genome and performed exploratory analyses on the raw datasets; M.E.S. and D.G.R. provided access to unpublished transcriptomic data and assisted in data mining and interpretation; R.C.L., R.C.H. and M.C. provided funding. All authors contributed to the drafting/editing of the manuscript.

\section{Competing interests}

The authors declare no competing interests. 


\section{Additional information}

Supplementary information is available for this paper at https://doi.org/10.1038/s41598-019-52840-z.

Correspondence and requests for materials should be addressed to F.O.H.

Reprints and permissions information is available at www.nature.com/reprints.

Publisher's note Springer Nature remains neutral with regard to jurisdictional claims in published maps and institutional affiliations.

(c) (i) Open Access This article is licensed under a Creative Commons Attribution 4.0 International License, which permits use, sharing, adaptation, distribution and reproduction in any medium or format, as long as you give appropriate credit to the original author(s) and the source, provide a link to the Creative Commons license, and indicate if changes were made. The images or other third party material in this article are included in the article's Creative Commons license, unless indicated otherwise in a credit line to the material. If material is not included in the article's Creative Commons license and your intended use is not permitted by statutory regulation or exceeds the permitted use, you will need to obtain permission directly from the copyright holder. To view a copy of this license, visit http://creativecommons.org/licenses/by/4.0/.

(c) The Author(s) 2019 